\title{
Optical Touch Pointer for Fluorescence Guided Glioblastoma Resection Using 5-Aminolevulinic Acid
}

Neda Haj-Hosseini, Johan Richter, Stefan Andersson-Engels and Karin Wårdell

\author{
Linköping University Post Print
}

Tweet

N.B.: When citing this work, cite the original article.

Original Publication:

Neda Haj-Hosseini, Johan Richter, Stefan Andersson-Engels and Karin Wårdell, Optical Touch Pointer for Fluorescence Guided Glioblastoma Resection Using 5-Aminolevulinic Acid, 2010, Lasers in Surgery and Medicine, (42), 1, 9-14.

http://dx.doi.org/10.1002/lsm.20868

Copyright: Wiley: 12 months http://eu.wiley.com/WileyCDA/

Postprint available at: Linköping University Electronic Press

http://urn.kb.se/resolve?urn=urn:nbn:se:liu:diva-53946 


\title{
Optical Touch Pointer for Fluorescence Guided Glioblastoma Resection Using 5-Aminolevulinic Acid
}

\author{
Neda Haj-Hosseini, MS, ${ }^{1}$ Johan Richter, MD, ${ }^{1,2}$ Stefan Andersson-Engels, $\mathrm{PhD},{ }^{3}$ and \\ Karin Wårdell, $\mathbf{P h D}^{1}$ \\ ${ }^{1}$ Department of Biomedical Engineering, Linköping University, Linköping 58185, Sweden \\ ${ }^{2}$ Department of Neurosurgery, Linköping University Hospital, Linköping 58185, Sweden \\ ${ }^{3}$ Department of Physics, Lund University, Lund 22100, Sweden
}

Background and Objective: Total tumor resection in patients with glioblastoma multiforme (GBM) is difficult to achieve due to the tumor's infiltrative way of growing and morphological similarity to the surrounding functioning brain tissue. The diagnosis is usually subjectively performed using a surgical microscope. The objective of this study was to develop and evaluate a hand-held optical touch pointer using a fluorescence spectroscopy system to quantitatively distinguish healthy from malignant brain tissue intraoperatively.

Study Design/Materials and Methods: A fluorescence spectroscopy system with pulsed modulation was designed considering optimum energy delivery to the tissue, minimal photobleaching of PpIX and omission of the ambient light background in the operating room (OR). 5 -aminolevulinic acid (5-ALA) of $5 \mathrm{mg} / \mathrm{kg}$ body weight was given to the patients with a presumed glioblastoma multiforme prior to surgery. During the surgery a laser pulse at $405 \mathrm{~nm}$ was delivered to the tissue. PpIX in glioblastoma tumor cells assigned with peaks at $635 \mathrm{~nm}$ and $704 \mathrm{~nm}$ was detected using a fiber optical probe.

Results/Conclusion: By using the pulsed fluorescence spectroscopy, PpIX fluorescence is quantitatively detected in the glioblastoma multiforme. An effective suppression of low power lamp background from the recorded spectra in addition to a significant reduction of high power surgical lights is achieved.

Key words: background light suppression, fluorescence spectroscopy; glioblastoma multiforme; intraoperative 


\section{Footnote:}

The authors certify that there is no affiliation with or financial involvement in any organization or entity with a direct financial interest in the subject matter or materials discussed in the manuscript (e.g., employment, consultancies, stock ownership, honoraria).

The study was supported by a group grant (311-2006-7661) by the Swedish Foundation for Strategic Research (SSF), Swedish Research Council (VR) and Swedish Governmental Agency for Innovation Systems (VINNOVA). 


\section{INTRODUCTION}

The highly malignant brain tumor, glioblastoma multiforme (GBM) is difficult to fully resect under direct vision in the operating field. The challenge is due to GBM's infiltrative way of growing and its morphological similarities to the surrounding functioning brain tissue. A higher extent of tumor resection is reported to be associated with a significant survival advantage [1]. Magnetic resonance and/or computed tomography images are taken before the surgery for observing the location and form of the tumor but still the important task of identifying tumor margins is based on intra-operative tissue palpation and visual inspection under the surgical microscope.

Fluorescence guidance using endogenous [2, 3] and exogenous [4, 5] types of fluorophores for brain tumor detection have previously been reported. Stummer and colleagues [6, 7] have evaluated a fluorescence microscopy system for guided resection of malignant gliomas. Protoporphyrin IX (PpIX) fluorescence induced by a dose (20 mg/kg bodyweight) of 5aminolevulinic acid (5-ALA) was used for intra-operative fluorescence microscopy guidance during the resection. However, utilization of fluorescence labeling and information is still far from the norm in clinical practice. Thus, further research and development of clinically suitable fluorescence methods is essential.

Our group previously used 5-ALA induced fluorescence and MR spectroscopy during stereotactic biopsies of human glioblastomas [8] and recently a first prototype of a fiber-optic based fluorescence spectroscopy system was introduced for guided neurosurgical tumor resection $[9,10]$. The significances of these studies are that the measurements are objective and a relatively low dose of ALA ( $5 \mathrm{mg} / \mathrm{kg}$ ) provokes detectable fluorescence peaks related to the malignant tumor tissue. This drug passes the leaky blood-brain barrier (BBB) in the tumor but not the intact BBB in normal brain tissue and is converted to the fluorescence marker PpIX in the malignant cells $[11,12]$. PpIX is a natural substance in the heme cycle which is rapidly eliminated from the body. Laser light at $405 \mathrm{~nm}$ is absorbed by protoporphyrin IX. The molecules of PpIX re-emit fluorescence with peaks at 635 and $704 \mathrm{~nm}$.

A major issue with fluorescence monitoring is the photobleaching of PpIX under light exposure. Therefore, a separate study was undertaken where the measurement parameters were optimized for obtaining minimal photobleaching effect during fiber optical measurements of fluorescence spectra [13]. Another problem in many applications involving optical measurements in the operating room is the superimposition of surrounding light sources including surgical lamps and microscope on the collected signal. The aim of the present study is to introduce the concept 
of an optical touch pointer for fluorescence guided resection of brain tumors. The system performance, and possibility to reduce surrounding light by system modulation, has been evaluated in the operating room during surgery.

\section{MATERIALS AND METHODS}

\section{System setup}

An optical touch pointer for on-line fluorescence spectroscopy measurements was set up (Fig. 1a). The components comprise a near-UV laser module with the maximal excitation light at $405 \mathrm{~nm}$ and maximal power of $50 \mathrm{~mW}$ (Oxxius, Lannion, France). The laser operates either in continuous or pulsed mode modulation. A spectrometer (EPP 2000, Stellarnet, Tampa, FL, USA) with a 2048 element CCD operating in the range of $240-850 \mathrm{~nm}$ wavelengths and with a resolution of $3 \mathrm{~nm}$ is used for light read out. To suppress the back-reflected light from reaching the spectrometer, a long pass, cutoff filter of $450 \mathrm{~nm}$ (Schott CG-GG-475-0.50-3, CVI Melles Griot, Albuquerque, NM, USA) is fixed in front of the detector slit of the spectrometer.

Excitation light is brought to the tissue through a hand-held fiber optical probe $\left(1_{\text {probe }}=12 \mathrm{~cm}\right.$, $\left.\varnothing=2 \mathrm{~mm}, 1_{\text {cable }}>4 \mathrm{~m}\right)$ having one transmitting fiber $\left(\varnothing_{\text {core }}=600 \mu \mathrm{m}, \varnothing_{\text {cladding }}=640 \mu \mathrm{m}\right.$, numerical aperture $=0.37)$ and nine receiving fibers $\left(\varnothing_{\text {core }}=200 \mu \mathrm{m}, \varnothing_{\text {cladding }}=240 \mu \mathrm{m}\right.$, numerical aperture $=0.22$ ). The receiving fibers are matched to fit the slit configuration of the detector. A miniature micro-positioner (OFR Inc., Caldwell, NJ, USA) was mounted at the interface of laser and fiber probe in order to allow the alignment of the laser light towards the fiber probe. The laser effect, pulse generation and its synchronization are controlled by a DAQcard (National Instruments, Inc., Austin, TX, USA) and software developed in LabVIEW ${ }^{\circledR}$ (Version 8, National Instruments, Inc., Austin, TX, USA). The different hardware components of the system are mounted in a compact box of $31 \times 25 \times 21 \mathrm{~cm}^{3}$ which together with a laptop is easily carried on a trolley to the operating room. The total weight of the box is $4.6 \mathrm{~kg}$.

\section{System performance}

The system was programmed to generate the pulses simultaneously with the spectrometer data collection. The laser pulse was set to have a constant duty cycle of $50 \%$ in order to enable a light spectrum collection followed by an equivalent dark spectrum collection. The dark spectrum is subtracted from the prior light spectrum giving a spectrum free from the background light effect (Equation 1).

Compensated spectrum (i) = Light spectrum (i) - Dark spectrum (i) Equation (1) 
The captured spectra are presented on the monitor in real time and saved for additional postprocessing. The on-line presentation allows for an immediate control of the signal quality and possibilities for adjustments on site.

The laser pulse width, which is set to be the same as the integration time of the spectrometer, was chosen as a trade off between the detectable amount of collected light, the minimal energy given to the tissue and minimal photobleaching of PpIX. Laser power $(0-30 \mathrm{~mW})$, tissue exposure time (range 0.06 - $65.50 \mathrm{~s}$ ) and the number of collected spectra can be changed through the software interface prior to initialization of a recording. Typical settings for a minimum of $2 \mathrm{~mJ}$ excitation at $405 \mathrm{~nm}$ allowing a distinguishable fluorescence signal during each spectrum, are $5 \mathrm{~mW}$ laser power and $0.4 \mathrm{~s}$ pulse width. Considering these time and power settings and that the probe is placed in contact with the tissue $\left(\mathrm{A}_{\text {exposed tissue }}=0.28 \mathrm{~mm}^{2}\right)$, the system gives an irradiance of $1.78 \mathrm{~W} / \mathrm{cm}^{2}$ and a fluence or dose of $0.71 \mathrm{~J} / \mathrm{cm}^{2}$ during each pulse.

The potential damage to the tissue by a laser beam occurs via tissue heating. Using Monte Carlo for light absorption and propagation, and a multiphysics program (COMSOL Multiphysics 3.4, Comsol AB, Stockholm, Sweden) for heat transfer simulation [14] the temperature increase was simulated using the optical and thermal parameters of brain $[15,16]$. The maximal temperature increase within each pulse $(5 \mathrm{~mW}, 0.4 \mathrm{~s})$ is estimated as $0.4^{\circ} \mathrm{C}$.

The system is wavelength-calibrated with the specific coefficients of the spectrometer provided by the manufacturer (Stellarnet, USA) and checked against the room's fluorescent lamp with known wavelengths for registration of intensity versus the correct wavelengths. An intensity calibration standard was produced from a steralizable plastic material with very stable fluorescence properties. A fluorescence spectrum of this calibration standard was recorded before each set of measurements.

\section{Evaluation of the system}

\section{Reduction of surrounding light}

The suppression of background light was tested in the operating room (OR). The lamps in the OR are categorized into low power (conventional fluorescent tube) and high power (surgical lamps; Hanualux, Siemens AG, Erlangen, Germany, and surgical microscope; OPMI Carl Zeiss $^{\odot}$, Oberkochen, Germany). Measurements were repeated $(n=3)$ with different pulse settings $(0.1-0.5 \mathrm{~s}$ and $1-10 \mathrm{~mW})$ on non-ALA treated human forearm skin on a volunteer and the fluorescent intensity standard. The distances between tissue and lamps were kept identical to the brain surgery circumstances. Five spectra were recorded with a pulse width of 
$0.4 \mathrm{~s}$ and excitation light of $5 \mathrm{~mW}$ using the fluorescent tube, surgical lamps and surgical microscope as a background light illumination, respectively. The integration time is set long enough to average out any fluctuations due to the $50 \mathrm{~Hz}$ drive current for the lamps.

\section{Measurements during tumor resection}

Patients undergoing surgical resection of presumed glioblastoma were given a $5 \mathrm{mg} / \mathrm{kg}$ bodyweight of 5-ALA dissolved in orange juice two hours prior to skull opening. They were then anesthetized as a preparation for surgery. The fiber probe used was sterilized with the STERRAD $^{\circledR}$ procedure. Measurements were approved by the local ethics committees (No: M139-07) and written informed consent was received from the patients. To allow direct comparability, measurements on one patient are presented in this study.

A measurement was performed on the calibration standard prior to the measurements on the brain as a primary check for the system operation. Measurements were then made on the surface of white, gray and known tumor tissue. Further recordings were made 1 - 2 mm inside the tumor as well as before and after resection on the tumor margin. In the latter cases biopsies were taken for pathological examination.

At each measurement site 5 spectra were collected. This was to allow further signal analysis in the primary measurements. During the collection of the spectra, the surgical microscope was directed away from the measurement field or its light was obstructed by hand.

\section{Data analysis}

The data were analyzed using MATLab 7.0.1 (MathWorks ${ }^{\mathrm{TM}}$, Inc., Natick, MA, USA). As the PpIX photobleaches quickly and considerably between the first recorded spectra [13], the averaging of the signals has been avoided. The distance and angle of the probe relative to the tissue are not perfectly reproducible in different measurements, in addition to the fact that the source power might be varied during different measurements. To overcome this challenge, the autofluorescence of each spectrum $(510 \mathrm{~nm})$ was taken as the reference level for the corresponding PpIX peak and a ratio of intensities, ratio number, was calculated for each compensated spectrum as explained by Equation 2. [17].

$$
\text { Ratio number of compensated spectrum }(i)=\left(\frac{I_{P p I X}-I_{\text {base }}}{I_{\text {autofl }}}\right)_{i} \quad \text { Equation (2) }
$$


where $\mathrm{I}_{\mathrm{PpIX}}$ is the PpIX fluorescence peak at $635 \mathrm{~nm}, \mathrm{I}_{\text {autofl }}$ is an average of the recorded signal around the peak which represents the peak for tissue autofluorescence, $I_{b a s e}$ is the autofluorescence at $635 \mathrm{~nm}$ and $i$ denotes the sequence number of spectrum (Fig. 2). Compensated spectrum (i) is obtained by subtracting dark spectrum (i) from the prior light spectrum (i), see Equation 1.

\section{RESULTS}

\section{Reduction of surrounding light}

The measurements on non-ALA treated forearm skin in the operating room with the background effect from fluorescent tubes, surgical lamps and the surgical microscope are presented in Fig. 3. The influence from the fluorescent tubes is effectively subtracted from the spectra (Fig. 3a). The influence from surgical lamps (Fig. 3b) and the surgical microscope (Fig. 3c) are reduced to a great extent.

\section{Measurements during tumor resection}

Measurments performed in healthy brain tissue, either white or gray, did not show any detectable sign of PpIX (Fig. 4a). Measurements performed on the tumor tissue showed a clear PpIX which is a sign of PpIX accumulation in the cancerous cells. Fig. $4 \mathrm{~b}$ shows a site measured inside the tumor where a high grade glioma was pathologically diagnosed. The ratio number for this spectrum is 6.0. Fig. 4c shows the signal from a site before and after resection. The surgeon did not review the spectroscopy results before announcing the diagnosis. Pathological biopsy at this location showed a grade III glioma and the ratio number was calculated to 2.1. After resection no PpIX fluorescence was recorded at this site. Blood at the probe interface creates a dip in the spectra at $580 \mathrm{~nm}$; however, a considerable amount of blood can block the total light propagation between the probe and the brain. In this case the probe was rinsed and the measurement was repeated. All the measurements presented in Fig. 4 were performed on the same patient.

\section{DISCUSSION}

An optical touch pointer using a fiber-optic based, fluorescence spectroscopy system with continuous and pulsed modulation was developed to detect the 5-ALA induced PpIX fluorescence in glioblastoma multiforme. The method and the developed technique are proven to optimally detect malignant tumor tissue in a short measurement time $(<1$ second for each 
measurement point). Conventional biopsies with examination conducted by a specialist in neuropathology are used as the gold standard for evaluation of the fluorescence spectroscopy diagnosis at specific measurement sites.

We have been able to effectively omit the interfering light from the fluorescence tubes in the operating room by using the pulsed modulation to subsequently detect light plus background and background only, allowing the background light to be accurately subtracted. The influence of the surgical lamp and microscope background was reduced to a great extent; however, as these lamps are of high power, a slight difference between the recording of the light and dark spectra due to both pulse width differences and probe movement may result in inaccurate spectra compensation. Such effects could be avoided in the recordings by directing the high power surgical light away from the measured tissue precisely before the measurement acquisition. All other light in the OR could remain the same without influencing the measurements in any critical way. Regarding the simulated temperature increase $\left(0.4^{\circ} \mathrm{C}\right)$ of exposed brain tissue during each pulse, it is not expected that in this specific application any clinical complication will arise due to laser heating. However, a long exposure time should be avoided.

What brings the use of this technique into clinical interest is its capability to quantitatively differentiate the malignant tissue from healthy tissue at the tumor margin. The device is easy to use and its design and construction are robust and easy to handle for the surgeon as well as for the nurses. In comparison to the well established ALA-based tumor detection system as developed by Stummer et al (with a filter integrated in operational microscopes), a disadvantage could be the fact that our device can not give an overall view of the operational field. It can be discussed to what extent this is necessary, as we do not intend to reinvent the basic neurosurgical operational techniques for these specific tumor resections. However, we believe that the optical probe could be a valuable complementary method to the microscope-based system. By using this technique, even low intensities of PpIX fluorescence can be detected. Also the probe can be inserted in the tumor to investigate the tumor's extent in depth.

An objective of future clinical studies is the correlation of the PpIX fluorescence intensity with the degree of tumor malignancy. Intensity of the PpIX fluorescence is primarily evaluated by the commonly used ratio of PpIX fluorescence to autofluorescence (ratio number). Although of diagnostic value, the ratio number is accompanied by a certain amount of error. Thus, further research into alternative analysis methods would be beneficial. A more precise control over the detection resolution by performing simulations and design of an optimum probe configuration 
is also of interest. Moreover, further research will include investigating PpIX uptake on the cellular level and estimation of sensitivity and specificity of the method within a larger group of patients.

In conclusion, a dual mode (continuous and pulsed) fiber-optic based, fluorescence spectroscopy system with the excitation light at $405 \mathrm{~nm}$ was developed. We were able to effectively detect 5-ALA induced PpIX fluorescence in the glioblastoma multiforme during surgical resection. The pulsed modulation of the system keeps the energy exposure to the tissue and the total measurement time short enough by synchronizing light delivery and fluorescence collection. A further benefit of this system is the omission of the background light from the lamps in the operating room.

\section{ACKNOWLEDGEMENTS}

The authors would like to thank the staff at the Neurosurgical Department of Linköping University Hospital. Technical support from research engineers at the Department of Biomedical Engineering at Linköping University, Per Sveider and Bengt Ragnemalm, in mechanical system assembly and consultation with Carina Fors (Lic. Eng., Linköping University) and Mikkel Brydegaard Sørensen (MSc., Lund University) with LabVIEW programming was of great value. This study was supported by the Swedish Governmental Agency for Innovation Systems (Vinnova), Swedish Foundation for Strategic Research (SSF) and Swedish Research Council (VR).

\section{REFERENCES}

1. Lacroix M, Abi-Said D, Fourney D R, Gokaslan Z L, Shi W, Demonte F, Lang F F, Mccutcheon I E, Hassenbusch S J, Holland E, Hess K, Michael C and Miller D, Raymond S. A multivariate analysis of 416 patients with glioblastoma multiforme: prognosis, extent of resection, and survival. Journal of neurosurgery 2001; 95: 190-198.

2. Andersson-Engels S, Elner Å, Johansson J, Karlsson SE, Salford LG, Strömblad LG, Svanberg K, Svanberg S. Clinical recording of laser-induced fluorescence spectra for evaluation of tumour demarcation feasibility in selected clinical specialities. Lasers in Medical Science 1991; 6(4):415-424.

3. Toms SA, Lin W-C, Weil RJ, Johnson MD, Jansen DE, Mahadevan-Jansen A. Intraoperative Optical Spectroscopy Identifies Infiltrating Glioma Margins with High Sensitivity. Neurosurgery 2005; 57(4):382-391.

4. Stummer W, Reulen H-J, Meinel T, Pichlmeier U, Schumacher W, Tonn J-C, Rohde V, Oppel F, Turowski B, Woiciechowsky C, Franz K, Pietsch T. Extent of resection and survival in glioblastoma multiforme: Identification of and adjustment for bias. Neurosurgery-online 2008; 62(3):564-576. 
5. Bogaards A, Varma; A, Collens SP, Lin A, Giles A, Yang VXD, Bilbao JM, Lilge LD, Muller PJ, Wilson BC. Increased brain tumor resection using fluorescence image guidance in a preclinical model. Lasers in Surgery and Medicine 2004; 35(3):181-190.

6. Stummer W, Novotny A, Stepp H, Goetz C, Bise K, Reulen HJ. Fluorescence-guided resection of glioblastoma multiforme by using 5-aminolevulinic acid-induced porphyrins: a prospective study in 52 consecutive patients. Journal of Neurosurgery 2000; 93: 1003:1013.

7. Stummer W, Pichlmeier U, Meinel T, Wiestler OD, Zanella F, and Reulen H-J. Fluorescence-guided surgery with 5-aminolevulinic acid for resection of malignant glioma: a randomised controlled multicentre phase III trial. The Lancet Oncology 2006; 7: 392-401.

8. Pålsson S. EO Backlund, O. Eriksson, P. Lundberg, Ö. Smedby, P. Sturnegk, K. Wårdell, K. Svanberg, and S. Andersson- Engels, ALA-PpIX fluoroscence and MR spectroscopy in connection with stereotactic biopsy of human glioblastomas, $\mathrm{In} \mathrm{PhD}$ thesis, Lund Institute of Technology, Sweden, 2003.

9. Ilias MA, Richter J, Westermark F, Brantmark M, Andersson-Engels S, and Wårdell K. "Evaluation of a fiber-optic fluorescence spectroscopy system to assist neurosurgical tumor resections. Proc. SPIE 2007; 6631: 66310W01-08.

10. Haj-Hosseini N, Andersson-Engels S, Wårdell K. Evaluation of a Fiber-Optic Based Pulsed Laser System for Fluorescence Spectroscopy. 14th Nordic-Baltic Conference on Biomedical Engineering and Medical Physics 2008; 363-366.

11. Novotny A, Stummer W. 5-Aminolevulinic Acid and the Blood-Brain Barrier - A Review. Medical Laser Application 2003; 18(1):36-40.

12. Moan J, Van Den Akker JTHM, Juzenas P, Ma LW, Angell-Petersen E, Gadmar ØB, Iani V. On the basis for tumor selectivity in the 5-aminolevulinic acid-induced synthesis of protoporphyrin IX. Journal of Porphyrins and Phthalocyanines 2001; 5(2):170-176.

13. Haj-Hosseini N, Richter J, Andersson-Engels S, Wardell K. Photobleaching behavior of protoporphyrin IX during 5-aminolevulinic acid marked glioblastoma detection. Proc. of SPIE. 2009; 7161: 716131-1-5.

14. Sturesson C, Andersson-Engels S. Mathematical modelling of dynamic cooling and pre-heating, used to increase the depth of selective damage to blood vessels in laser treatment of port wine stains. Physics in Medicine and Biology 1996; 41(3):413-428.

15. Schwarzmaier H-J, Eickmeyer F, Fiedler VU, Ulrich F. Basic Principles of Laser Induced Interstitial Thermotherapy in Brain Tumors. Medical Laser Application 2002; 17(2):147-158.

16. Yaroslavsky AN, Schulze PC, Yaroslavsky IV, Schober R, Ulrich F, Schwarzmaier HJ. Optical properties of selected native and coagulated human brain tissues in vitro in the visible and near infrared spectral range. Physics in medicine and biology 2002; 47(12):2059-2073.

17. Bendsoe N, Persson L, Johansson A, Axelsson J, Svensson J, Grafe S, Trebst T, Andersson-Engels S, Svanberg S, Svanberg K. Fluorescence monitoring of a topically applied liposomal Temoporfin formulation and photodynamic therapy of nonpigmented skin malignancies. J Environ Pathol Toxicol Oncol 2007; 26(2):117-126. 


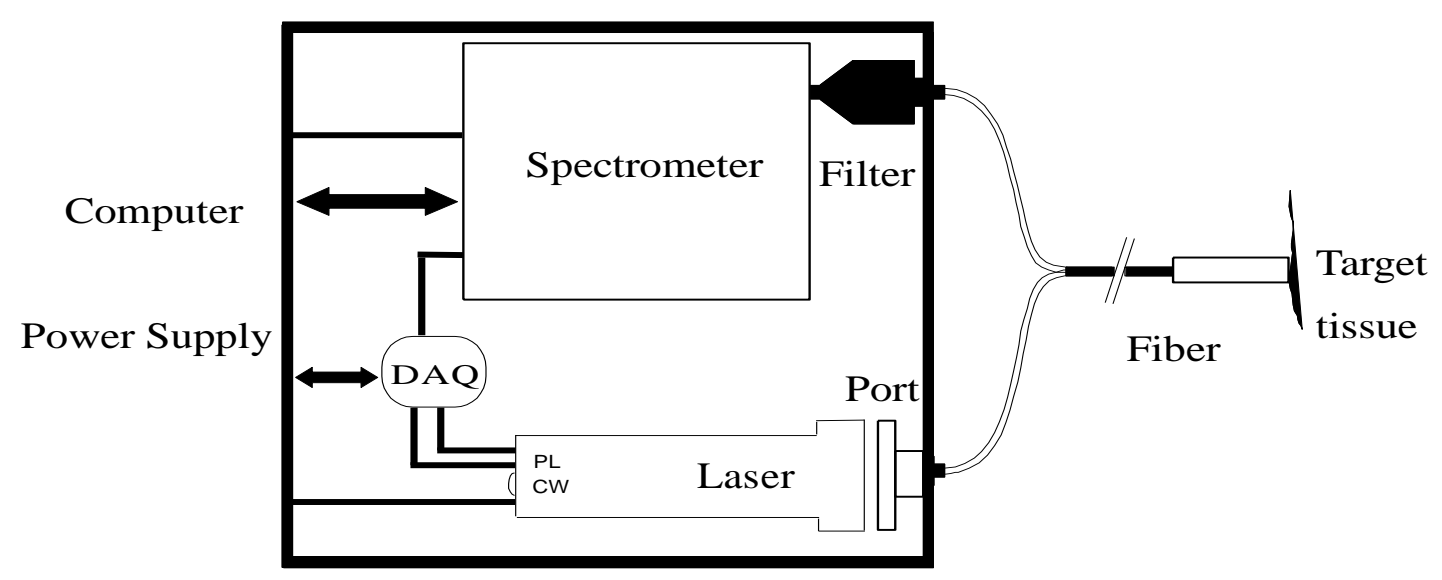

(a)

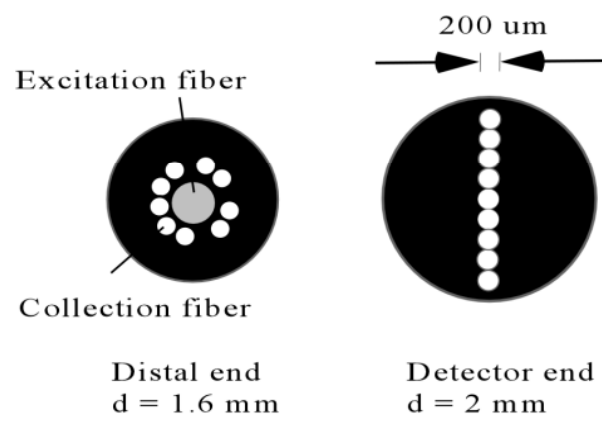

(I)

(II)

(b)

Fig. 1. (a) Hardware design of fiber-optic based, pulsed system. (b) Fiber probe configuration at (I) probe tip, and (II) detector end. The vacant parts are for the placement of fiber optics of different optical diagnostic applications. The excitation fiber and the surrounding collection fibers, including the cladding, have a diameter of 0.64 and $0.24 \mathrm{~mm}$, respectively. 


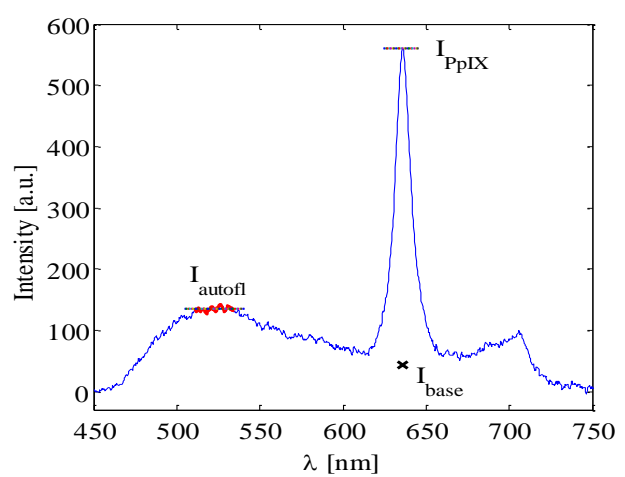

Fig. 2. A typical compensated fluorescence spectrum measured on tumor illustrating fluorescence intensity in arbitrary units versus wavelength. Peaks at 635 and $704 \mathrm{~nm}$ are representative of PpIX. The peak at $510 \mathrm{~nm}$ is due to the brain tissue autofluorescence. Ratio number (Equation 2) of the PpIX in this figure is 3.8.
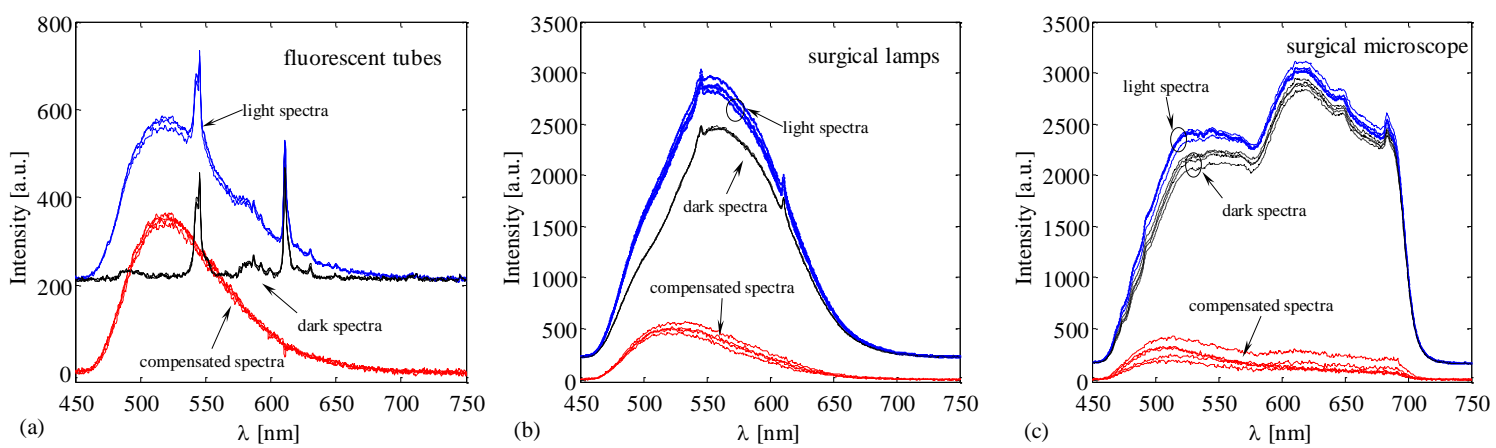

Fig. 3. Light, dark and the compensated spectra from measurements under lamps in the operating room. All measurements were performed on skin under $5 \mathrm{~mW}$ laser power and $0.4 \mathrm{~s}$ exposure time. 


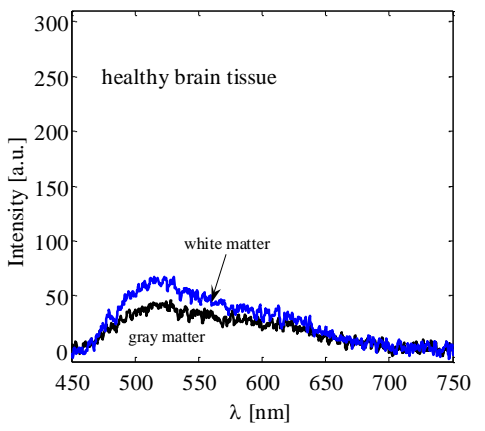

(a)

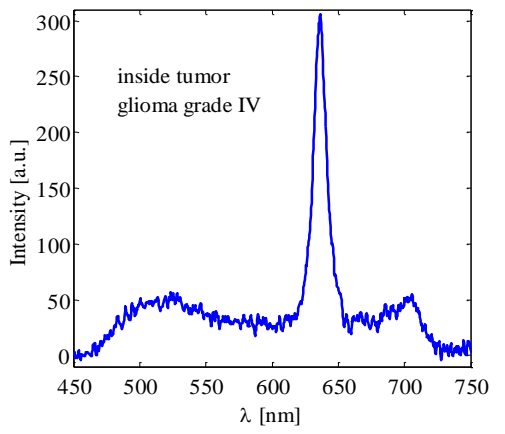

(b)

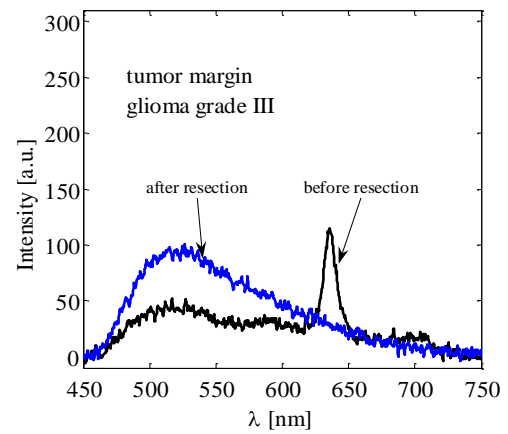

(c)

Fig. 4. Measurements performed with the pulsed system using a pulse width of $0.4 \mathrm{~s}$ and $5 \mathrm{~mW}$ power at $405 \mathrm{~nm}$ on one patient with glioblastoma multiforme. Fluorescence spectra from (a) healthy white and gray matter in vicinity of the tumor, (b) inside the tumor where pathological biopsy showed glioma grade IV, and (c) before and after resection of a site at tumor margin where pathological biopsy showed a glioma grade III. Ratio numbers for (b) and (c) are 6.0 and 2.1 , respectively. 